\title{
Tangence
}

\section{Archives, de Paul-Émile Saulnier ou « L'incarcération des images ». Galerie d'art de l'UQAR - 23 mars-3 avril 1994}

\section{Élisabeth Haghebaert}

Numéro 44, juin 1994

La référence littéraire

URI : https://id.erudit.org/iderudit/025819ar

DOI : https://doi.org/10.7202/025819ar

Aller au sommaire du numéro

Éditeur(s)

Tangence

ISSN

0226-9554 (imprimé)

1710-0305 (numérique)

Découvrir la revue

Citer ce document

Haghebaert, É. (1994). Archives, de Paul-Émile Saulnier ou « L'incarcération des images ». Galerie d'art de l'UQAR - 23 mars-3 avril 1994. Tangence, (44),

129-133. https://doi.org/10.7202/025819ar d'utilisation que vous pouvez consulter en ligne.

https://apropos.erudit.org/fr/usagers/politique-dutilisation/ 


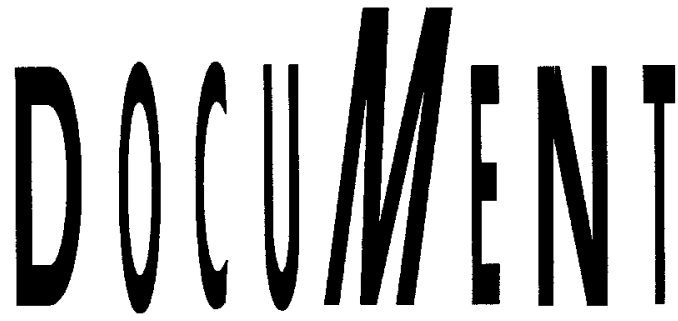

\section{Archives, de Paul-Émile Saulnier ou "L'incarcéra- tion des images". Galerie d'art de I'UQAR - 23 mars-3 avril 1994}

Paul-Émile Saulnier est un artiste doublé d'un aventurier. Preuve en est l'exposition qui se tenait du 23 mars au 3 avril à la Galerie d'art de l'UQAR. Familier des grands espaces à la mesure de ses sculptures-installations - monuments expiatoires des atrocités du monde - dont Moncton et Montréal, ainsi que le Musée de Rimouski entre autres, avaient été les hôtes, il n'a pas hésité ici à explorer une autre dimension en relevant le défi de l'exiguité, sans renoncer à sa démarche personnelle de réappropriation de l'Histoire. Force est de constater que la concession à un espace restreint, style récession, n'aura été qu'un compromis, car se retrouvaient réinvestis là, même à l'échelle miniature, la dimension colossale de l'entreprise, son foisonnement et sa généreuse prolixité. Ennemi des solutions sélectives et fidèle à son esthétique de la saturation, Saulnier a obéi à la contrainte en homme de son temps sur le mode postmoderne du recyclage et de l'archivage virtuel.

Résultat: un musée imaginaire de pocbe, sorte de "mise en boîte " ou d' "incarcération " autoreprésentative et citationnelle constituée de fragments de tout ce qui témoigne de l'œuvre. Photos, affiches, cartons d'invitation, toutes sortes d'images "ready made" réunies sous le signe du trucage forment un lieu de mémoire et de fusion des genres et des matériaux. Clin d'œil aux maitres que sont par exemple Rubens, de la Tour, Goya ou Delacroix, des extraits de chromos d'œuvres célèbres plus ou moins "trafiquées "s'y intègrent aussi bien que - vestiges d'installations ou simple séduction plastique des objets et de la matière brute - 
130

des fragments du réel tels que bois, verre, clous, boulons et plexiglas.

Sous le régime de la récupération et de l'allusion donc, ce sanctuaire d'images issu d'un travail de "gullivérisation" condense bel et bien vingt ans de production en une soixantaine de reliquaires métalliques longilignes hiératiquement alignés, comme les touches d'un clavier, à hauteur du regard. Leur format identique et leur aspect sériel contribuent simultanément à un effet de répétition et de variation. Toute monotonie est en effet exclue par le jeu discret de l'implicite, c'est-à-dire les affinités qui, ici et là, forment diptyques ou triptyques, les intervalles entre les cadres, la couleur, voire l'épaisseur de ceux-ci, ou encore la soudaine horizontalité de quelques-uns de ces tableaux-montages à part. Bref, la sobriété intense qui résulte de l'apparente économie des moyens invite le visiteur à observer en détails cet "étalage" en bidimension, ainsi qu'à concentrer son attention sur la qualité réflexive de l'œuvre, qu'il s'agisse de spéculations sur le spéculaire et le spectaculaire, ou d'interrogations sur la valeur de la représentation de toute forme de référence.

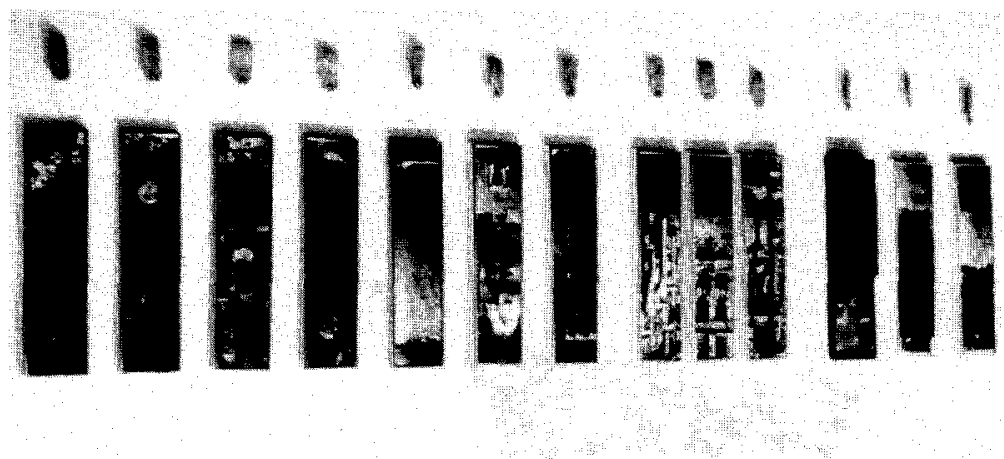

Archives: le titre dénonce le propos classificatoire de l'exposition. On s'attend à du stockage, mais ne pas s'y méprendre: l'ordonnancement qui en résulte obéit à une logique associative plutôt qu'à des relations rationnelles. Tentative de mise en ordre du passé, les étiquettes au bas de chaque tableau rappellent à qui veut l'entendre le dérisoire de l'entreprise. $\mathrm{Y}$ voir donc matière à 
simulacre plutôt que des titres au sens ordinaire du terme. D'ailleurs la reversibilité des textes qui s'y inscrivent recto verso, comme autant de bribes arrachées à la matière brute du langage, est un indice de la logique aléatoire à laquelle ils obéissent.

Coups d'envoi, constats, consignes ou "obsessions", ces phrases incitent le lecteur à poursuivre le poème ou la rêverie qu'ils amorcent. "Traquer les idées, la page est un piège", "La nuit s'est déportée", "Intolérable intolérance", "Les trains partaient au crépuscule", "Cartel de la peur": ces mots d'ordre rappellent le caractère dramatique de l'ouvre de Saulnier et le théâtral sens de la mise en scène dont témoignaient ses installations, depuis Les nuits de vitres et La nuit des masques (opéra), jusqu'à Un siècle de cendre. Dénonçant l'oppression sous toutes ses formes, comme l'ont fait avant lui Goya ou Daumier, celui-ci convoque le visiteur dans un univers de ténèbres lourd de sens et de menaces et, qu'il soit carcéral ou concentrationnaire, l'obscurité y tient lieu de matière et d'objet.

Sous cette apparente absence de couleurs qui semble faite de particules de suie, de cendre ou de carbone, l'oil qui ne craint pas de scruter l'étrange pénètre dans le monde mystérieux des métamorphoses où des colonnes s'enracinent. Spéléologue, il y découvre la dimension cachée de ce qui se trame autant sous les lames de plancher que sous les lieux communs. Ce qui se défait, se délite, ce qui échappe à l'entendement, ce qu'on ne voit pas ou ce qu'on tait: l'incongru à la Beckett, le silence du cri de Munch, l'inframonde des petits riens qui changent tout, bref, tout ce que captent et reflètent, comme les tropismes de Sarraute, les boîtes à images de Saulnier.

En fait, cet inventaire iconographique se double d'une exploration qui tire parti du statut factice des reproductions. Partant du principe qu'il s'agit de faux, l'artiste peut laisser libre cours à sa fantaisie et rassembler, combiner, réactiver et reformuler les différentes strates de l'œuvre, qu'elles soient déjà-vues, oubliées ou inédites, en brouillant les parcours et les pistes sans vergogne. La réassomption de l'œuvre antérieur passe par cette sorte de retour au chaos que provoquent la remise en cause des compositions figées et la déstructuration des agencements établis, et bien malin qui s'y retrouve entre les fragments de caricatures, d'affiches, de photos ou de dessins. Comme dans le rêve, les souvenirs un instant entrevus se perdent dans de nouvelles configurations. 
D'autre part, on comprend avec quelle jubilation ce "trafic" (comme le désigne Saulnier) s'érige en système. Transposé à l'histoire de l'art, il permet de fracturer la routine et de satisfaire sans scrupules un fantasme iconoclaste d'appropriation de l'héritage culturel. Ainsi, habiller de puritanisme fin de siècle quelques Grâces de Rubens, frapper d'un matricule une scène d'exode biblique et assigner au Massacre de Scio ou aux Désastres de la guerre une fonction décorative ou anecdotique pour finalement les insérer dans sa propre production instaure une pratique transgressive qui rappelle le "maghanage "littéraire que Ducharme a fait subir au Cid de Corneille.

Séquelles du surréalisme ou du "plaisir gamin de choquer", ces téléscopages disons néoculturels ont, tout en rendant un hommage oblique aux grands maîtres du passé, l'avantage de faire surgir une sorte d'outre-sens, comme si mutiler les images pour en "cracher" d'autres ("par mauvaise humeur", lit-on sur une étiquette) que l'on pourra à leur tour "crasher" et même "scratcher" pouvait annihiler ou du moins exorciser les cauchemars qui hantent la conscience. C'est ce que semblent démontrer en particulier, comme autant d'intrusions ou d'aincarcérations" colorées, quatre médaillons qui proposent un décryptage à la loupe de citations picturales de Delacroix et de Georges de la Tour, offrant ainsi le plus bel exemple d'anamorphose qui soit. Bref, on le voit, ces Archives sont loin d'être classées, leur dimension réflexive laisse présager des développements futurs risquant d'outrepasser les limites de la lilliputienne galerie qui les a fait s'exhumer. 


\section{Élisabeth Haghebaert}

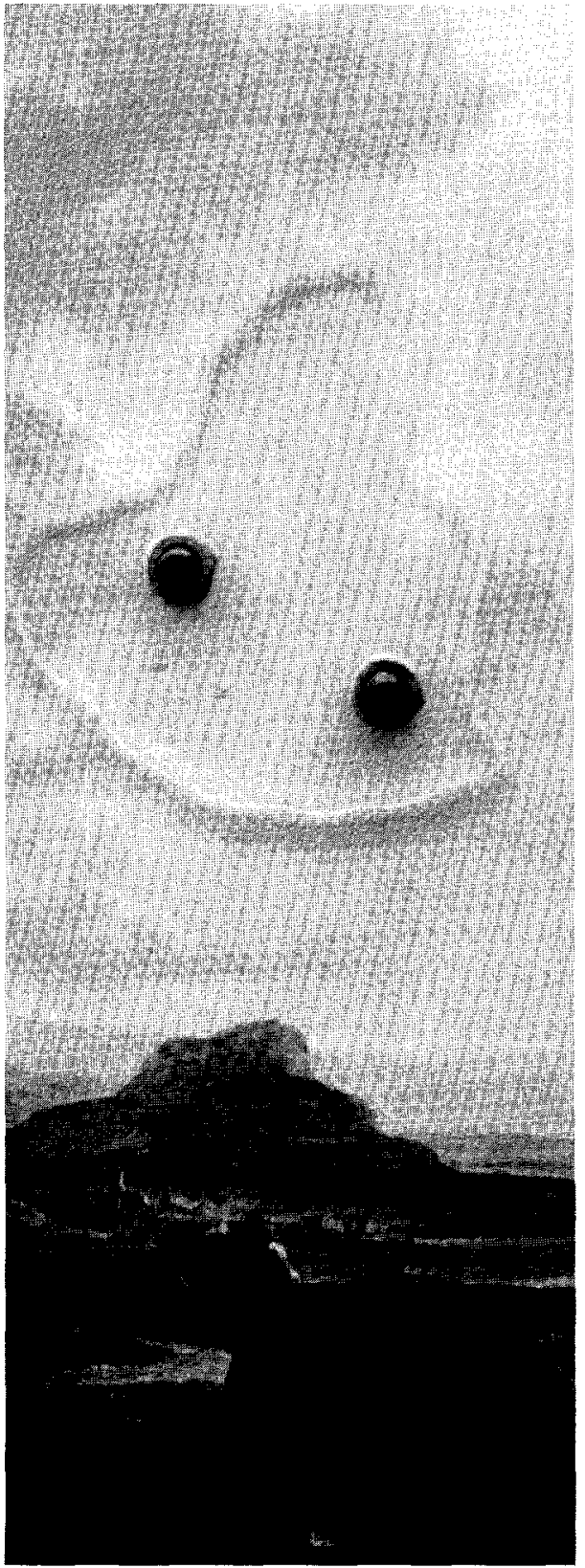

